\title{
EFEITO DA TERAPIA NUTRICIONAL NA QUALIDADE DE VIDA DE PACIENTES DIABÉTICOS TIPO 2
}

Joana Ribeiro Colombo

Barbisan ${ }^{1}$ Nutricionista.

Mestranda do Programa de PósGraduação em Ambiente e Saúde da Universidade do Planalto Catarinense - UNIPLAC. E-mail:

joanacbarbisan@uniplaclages.edu.br

\section{Michael Ramos Nunes ${ }^{2}$}

Químico. Doutor em Química. Docente do Instituto Federal de Santa Catarina-IFSC Campus Lages. E-mail:

michael.nunes@ifsc.edu.br

\section{Elisa Pazzinato Telli ${ }^{3}$}

Biomédica. Mestre em Bioquímica e Biologia Molecular. Docente da UNIPLAC. E-mail:

elisatelli@uniplaclages.edu.br

\section{Melissa Kaiser ${ }^{4}$}

Farmacêutica e Bioquímica. Mestre

em Farmácia. Docente

UNIFACVEST. E-mail:

melissinhakayser@hotmail.com

Natalia Veronez da Cunha ${ }^{5}$ Fisioterapeuta. Doutora em Fisiologia. Docente Programa de Pós-Graduação em Ambiente e

Saúde da Universidade do Planalto Catarinense-UNIPLAC. E-mail: nat_cunha@uniplaclages.edu.br

\section{Cleonice Gonçalves da}

Rosa ${ }^{6}$ Química de Alimentos. Doutora em Ciência dos Alimentos. Pós-doutoranda do Programa de Pós-Graduação em Ambiente e Saúde da Universidade do Planalto Catarinense-UNIPLAC. E-mail: cleorosaqm@yahoo.com.br

\author{
EFFECT OF NUTRITIONAL THERAPY ON THE QUALITY OF \\ LIFE OF TYPE 2 DIABETIC PATIENTS
}

\section{RESUMO}

Para prevenir o diabetes tipo 2 (DM2) ou as suas complicações, existe muito interesse na identificação de alimentos vegetais com propriedades hipoglicêmicas para auxiliar no tratamento do DM2. O objetivo desse trabalho foi avaliar o efeito de uma terapia nutricional não convencional nos índices de glicemia de jejum e nos parâmetros de qualidade de vida em pacientes com DM2. Foi conduzido um ensaio clínico randomizado com 23 pacientes com DM2, divididos entre Grupo controle e Grupo intervenção com araçá-vermelho. Os indivíduos do grupo intervenção ingeriram $50 \mathrm{~g} /$ dia da polpa dos frutos de araçá por 60 dias, enquanto para o grupo controle a ingestão de água foi considerada como tratamento controle. Os níveis de glicemia de jejum foram avaliados no período basal e após 60 dias. Foram aplicados dois instrumentos, um questionário sociodemográfico e de avaliação clínica e um de qualidade de vida na versão brasileira do Diabetes Quality of Life Measure - DQOL-Brasil. Os pacientes que consumiram as polpas de araçá-vermelho reduziram significativamente os parâmetros glicêmicos: $25 \%$ nos níveis de glicose de jejum $(p<0,05)$, e foi identificado um efeito positivo entre o consumo de araçá vermelho, melhora nos níveis glicêmicos e melhor qualidade de vida relatada. Os resultados indicaram impacto positivo no consumo regular de araçá vermelho sobre os níveis de glicemia que podem contribuir para a saúde do sistema cardiovascular, e reduzir o risco de desenvolver complicações pelo DM2

PALAVRAS-CHAVE: Compostos Bioativos. Diabetes tipo 2. Qualidade de vida 


\section{ABSTRACT}

To prevent type 2 diabetes (DM2) or its complications, there is much interest in identifying plant foods with hypoglycemic properties to help treat DM2. The aim of this study was to evaluate the effect of an unconventional nutritional therapy on fasting glucose levels and quality of life parameters in patients with DM2. A randomized clinical trial was conducted with 23 patients with $\mathrm{DM} 2$, divided into a control group and an intervention group with red stingray. Individuals in the intervention group ingested $50 \mathrm{~g} /$ day of araçá fruit pulp for 60 days, while for the control group, water intake was considered as a control treatment. Fasting blood glucose levels were assessed at baseline and after 60 days. Two instruments were applied, a sociodemographic and clinical assessment questionnaire and a quality of life questionnaire in the Brazilian version of the Diabetes Quality of Life Measure - DQOL-Brazil. Patients who consumed the red macaw pulp significantly reduced glycemic parameters: $25 \%$ in fasting glucose levels $(p<0.05)$, and a positive effect was identified between the consumption of red macaw, improvement in blood glucose levels and better quality of life reported. The results indicated a positive impact on the regular consumption of red macaw on blood glucose levels that can contribute to the health of the cardiovascular system, and reduce the risk of developing complications from DM2.

KEYWORDS: Bioactive compounds. Type 2 diabetes. Quality of Life.

\section{INTRODUÇ̃̃O}

A Sociedade Brasileira de Diabetes (SBD, 2016), classifica o Diabetes Mellitus (DM) em diferentes categorias clínicas: DM tipo 1, DM tipo 2, DM gestacional e outros tipos específicos de DM. Além das referidas classificações já citadas, são encontradas mais duas categorias, chamadas de pré-diabetes, que são a glicemia de jejum alterada e a tolerância à glicose diminuída (SBD, 2016).

O diabetes impacta significativamente a vida dos pacientes, seja diretamente ou por causa de suas complicações, afetando negativamente os principais componentes da qualidade de vida (QV) (TRIKKALINOU, PAPAZAFIROPOULOU, MELIDONIS; 2017). Segundo a Organização Mundial de Saúde (THE WHOQOL GROUP, 1995), a qualidade de vida relacionada à saúde é um conceito amplo, percebido pelo indivíduo e afetado de uma forma complexa pela saúde física, estado psicológico, nível de independência, relações sociais, crenças pessoais e a relação com as características do seu ambiente.

A maioria dos casos de DM encontrados são do tipo 2 (DM2). Esse distúrbio aparece quando o organismo não consegue usar adequadamente a insulina que produz, ou não produz insulina suficiente para controlar a taxa de glicemia. Este último é o caso mais comum, afetando cerca de $90 \%$ das pessoas com diabetes. Dependendo da gravidade, ele pode ser controlado com atividade física e planejamento alimentar. Em outros casos, exige o uso de insulina e/ou outros medicamentos para controle da glicose (SBD, 2016).

Por conta do aumento acelerado no número de casos de diabetes, diversas pesquisas são realizadas com a finalidade de identificar plantas e frutos com propriedades hipoglicêmicas ou anti-hiperglicêmicas e que possam auxiliar no tratamento ou prevenção do diabetes (SALAU et al., 2020, PEREIRA et al., 2018). As terapias nutricionais não convencionais podem ser utilizadas para reduzir os índices glicêmicos dos alimentos e, consequentemente, a 
hiperglicemia, com o interesse de reduzir o risco de DM2 (PEREIRA et al., 2018). Dentre as várias espécies de plantas e frutos pesquisadas, os frutos de Araçá vermelho (Psidium cattleyanum Sabine), espécie vegetal nativa do Sul do Brasil, podem apresentar ações benéficas para os pacientes com DM2 (PEREIRA et al., 2018). Estudos realizados in vitro demonstraram que os frutos de Psidium cattleyanum Sabine reduzem a formação de produtos de glicação avançada, substâncias formadas a partir da oxidação de açúcares e gorduras, e que estão envolvidas no desenvolvimento das complicações crônicas do DM2 (PEREIRA et al., 2018). Neste contexto, o objetivo desse estudo foi conhecer o efeito do consumo de frutos de araçá na qualidade de vida de pacientes com DM2.

\section{MATERIAL E MÉTODOS}

Foi realizado um estudo intervencional através de um ensaio clínico, de tratamento, randomizado-controlado, em um período de 60 dias. A população do estudo foi composta por 22 indivíduos, de ambos os sexos, com idade média de 61,10 \pm 6,7 anos, usuários de uma Unidade de Saúde Básica-UBS no município de Lages-SC, selecionados de forma intencional e previamente diagnosticados com DM2. O estudo foi aprovado pelo Comitê de Ética em Pesquisa da Universidade do Planalto Catarinense - UNIPLAC (registrado sob o no. 2.620.370). Todos os participantes assinaram o consentimento para participação na pesquisa. O ensaio clínico foi registrado no RBR- 77bg2m.

Os pacientes foram divididos de forma aleatória em 2 grupos, grupo controle, com 6 mulheres e 3 homens e grupo intervenção composto por 11 mulheres e 2 homens. A ingestão de água foi considerada como tratamento controle. O grupo intervenção fez o consumo diário de $50 \mathrm{~g}$ das polpas de araçá vermelho em 30 $\mathrm{mL}$ de água por um período de 60 dias. As amostras de sangue venoso periférico foram coletadas de todos os participantes (grupo controle e intervenção), após um jejum de 12 horas, no período basal e após 60 dias do consumo das polpas das frutas. Foram avaliados os níveis plasmáticos de glicose de jejum dos pacientes por método enzimático, usando as enzimas glicose-oxidase/peroxidase em equipamento automatizado (Siemens Healthcare Diagnostic, EUA). Os instrumentos aplicados foram: questionário sociodemográfico e de avaliação clínica, e um segundo de qualidade de vida na versão brasileira do Diabetes Quality of Life Measure - DQOL-Brasil (CORRER et al. 2008).

Os dados quantitativos estão apresentados na forma de média e desvio padrão, e os dados categóricos estão apresentados na forma de frequência absoluta e relativa. Os dados foram submetidos ao teste de normalidade de Shapiro-Wilk, e comparados no intragrupos pelo teste t pareado e no intergrupo pelo teste $t$ independente não pareado, utilizando o programa Prism versão 8.0.

\section{RESULTADO E DISCUSSÃO}

\section{CARACTERÍSTICAS SOCIODEMOGRÁFICAS CLÍNICAS DOS PARTICIPANTES}

$\mathrm{Na}$ avaliação sociodemográfica, percebeu-se maior diferença entre os grupos controle e intervenção nas variáveis: estado civil, gênero, raça e ocupação. Quando comparados, o grupo intervenção apresentou a maioria dos indivíduos composto de mulheres casadas, de raça branca, autônomas, 
desempregadas ou do lar. Os resultados sociodemográficos estão demonstrados na Tabela 1.

Tabela 1 - Perfil sociodemográfico dos participantes.

\begin{tabular}{|c|c|c|c|c|}
\hline \multicolumn{2}{|c|}{ Variáveis Sociodemográficas } & $\begin{array}{c}\text { Individuos } \\
(\mathrm{n}=22) \\
(\%)\end{array}$ & \multirow{2}{*}{$\begin{array}{c}\text { Controle } \\
(\mathbf{n}=9) \\
(\%)\end{array}$} & \multirow{2}{*}{ 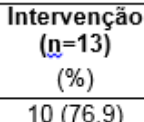 } \\
\hline \multirow{3}{*}{ Estado civil } & Casado & $15(682)$ & & \\
\hline & Divorciado & $4(18,2)$ & $3(33,3)$ & $1(7,7)$ \\
\hline & Viúvo & $3(8,3)$ & $1(11,1)$ & $2(15,4)$ \\
\hline \multirow{2}{*}{ Gênero } & Feminino & $18(81,8)$ & $6(66,7)$ & $12(92,3)$ \\
\hline & Masculino & $4(18,2)$ & $3(33,3)$ & $1(7,7)$ \\
\hline \multirow{3}{*}{ Raça } & Branco & $16(72,7)$ & $4(44,4)$ & $12(92,3)$ \\
\hline & Negro & $2(9,1)$ & $2(22,2)$ & $0(0,0)$ \\
\hline & Pardo & $4(18,2)$ & $3(33,3$ & $1(7,7)$ \\
\hline \multirow{3}{*}{ Escolaridade } & Analfabeto & $2(9,5)$ & $1(12,5)$ & $1(7,7)$ \\
\hline & Ensino Fundamental incompleto & $16(76,2)$ & $6(75)$ & $10(76,9)$ \\
\hline & Ensino Médio incompleto & $3(14,3)$ & $1(12,5)$ & $2(15,4)$ \\
\hline \multirow{5}{*}{$\begin{array}{l}\text { Ocupação } \\
\text { Profissional }\end{array}$} & Do Lar & $7(31,8)$ & $2(22,2)$ & $5(38,5)$ \\
\hline & Aposentado & $9(72,7)$ & $6(66,7)$ & $3(23,1)$ \\
\hline & Autônomo & $2(9,1)$ & $0(0,0)$ & $2(15,4)$ \\
\hline & Comerciário & $1(4,5)$ & $1(11,1)$ & $1(7,7)$ \\
\hline & Desempregada & $3(13,6)$ & $0(0,0)$ & $2(15,4)$ \\
\hline
\end{tabular}

Com relação às características clínicas dos pacientes (Tabela 2), a maioria apresentou diagnostico de DM2 pelo menos há 5 anos, histórico familiar em $1^{\circ}$ grau para DM2. E além do diagnostico de DM2 também possuía diagnostico para Hipertensão arterial, níveis alterados de colesterol, doença arterial coronariana (DAC), sedentarismo, estresse e consumo inadequado de água por dia.

Dentre os fatores de risco do DM2, a literatura cita como mais prevalentes o sedentarismo, seguido pelo excesso de peso, obesidade central, glicemia plasmática de jejum elevada ( $\geq 100 \mathrm{mg} \cdot \mathrm{dl}-1)$, hipertensão arterial (> 140/90 $\mathrm{mmHg}$ ), hábitos alimentares inadequados, avanço da idade ( $>45$ anos), histórico familiar de diabetes, HDL-colesterol baixo, <35 mg.dL-1, e/ou triglicerídeos elevados, >150 mg.dL-1, história de diabetes gestacional, diagnóstico prévio de síndrome de ovários policísticos e doença cardiovascular, cerebrovascular ou vascular periférica (LIMA, et al. 2014). 
Tabela 2- Perfil clinico dos pacientes.

\begin{tabular}{|c|c|c|c|}
\hline Fatores de rlaco & $\begin{array}{c}\text { Indlviduos } \\
(n=22)\end{array}$ & $\begin{array}{c}\text { Controle } \\
(n=9)\end{array}$ & $\begin{array}{c}\text { Intervençao } \\
(n=13)\end{array}$ \\
\hline Dlagndatico de DM2 [\%] & $22(100)$ & $9(100)$ & $13(100)$ \\
\hline \multicolumn{4}{|l|}{ Dlagndatico de DM (anos) } \\
\hline$<1$ ano & $4(18,2)$ & $0(0,0)$ & $4(30,8)$ \\
\hline $1-5$ anos & $14(38,9)$ & $6(66,7)$ & $8(61,5)$ \\
\hline $6-10$ anos & $2(9,1)$ & $1(11,1)$ & $1(7,7)$ \\
\hline$>10$ anos & $2(9,1)$ & $4(22,2)$ & $0(0,0)$ \\
\hline Hlatdrico famlllar de DM (\%) & $14(63,6)$ & $6(66,7)$ & $8(61,5)$ \\
\hline \multicolumn{4}{|l|}{ Grau de Parentesco [\%] } \\
\hline $1^{*}$ Grau & $15(68,2)$ & $8(88,9)$ & $7(53,8)$ \\
\hline $2^{*}$ grau & $1(4,5)$ & $0(00)$ & $1(7,7)$ \\
\hline Outros & $6(16,7)$ & $1(33,3)$ & $5(38,5)$ \\
\hline Medicaçào DM [\%] & $18(81,8)$ & $7(77,8)$ & $11(84,6)$ \\
\hline Dlagndatico de HAS [\%] & $17(77,3)$ & $8(88,9)$ & $9(69,2)$ \\
\hline Dlagndatico de Coleaterol (\%) & $12(54,5)$ & $3(33,3)$ & $9(69,2)$ \\
\hline Dlagndatico de Trigllcedes (\%) & $9(40,9)$ & $2(22,2)$ & $7(53,8)$ \\
\hline Tabaglita (\%) & $7(31,9)$ & $2(22,2)$ & $5(38,5)$ \\
\hline Hlatorico famlliar de DAC (\%) & $14(63,6)$ & $6(66,7)$ & $8(61,5)$ \\
\hline Dlagndatico de Prob. Resp. (\%) & $6(27,3)$ & $5(55,6)$ & $1(7,7)$ \\
\hline Dlagndatico de Prob. Hepatico (\%) & $8(36,4)$ & $5(55,6)$ & $3(23,1)$ \\
\hline Dlagndatico de Prob. Renal (\%) & $3(13,6)$ & $2(22,2)$ & $1(7,7)$ \\
\hline Dlagndatico de Hlpertireolde (\%) & $4(18,2)$ & $2(22,2)$ & $2(15,4)$ \\
\hline Dlagndatico de Hipotireolde (\%) & $0(0,0)$ & $0(0,0)$ & $\sigma(0,0)$ \\
\hline Proceaso Infecclo:olinflamatorio (\%) & $6(27,3)$ & $4(44,4)$ & $2(15,4)$ \\
\hline Ativo Flaicamente (\%) & $7(31,8)$ & $3(33,3)$ & $4(30,8)$ \\
\hline Consumo de Beblda alcodilca (\%) & $0(0,0)$ & $0(0,0)$ & $7(53,8)$ \\
\hline Eatresae ("/4) & $14(63,6)$ & $7(77,8)$ & $7(53,8)$ \\
\hline Hlitdrico de Infarto Mlocardlo [\%] & $2(9,1)$ & $1(11,1)$ & $1(7,7)$ \\
\hline Alteraçరөs gastrolnteatinala (\%) & $13(59,1)$ & $7(77,8)$ & $6(46,2)$ \\
\hline Evacuaçao dlarla (\%) & $18(81,8)$ & $8(88,9)$ & $10(76,9)$ \\
\hline Consumo de agua - 2 L/dla (\%) & $6(27,3)$ & $3(33,3)$ & $3(23,1)$ \\
\hline Presença de Alergla Allmentar (\%) & $3(13,6)$ & $3(33,3)$ & $0(0,0)$ \\
\hline
\end{tabular}

Fonte: autora propra

Dentre as complicações macrovasculares ocasionadas pelo DM2, a doença arterial coronariana é considerada a complicação mais importante e a principal causa de morte entre os diabéticos adultos, sendo detectada em mais de 50\% dos indivíduos com DM2 (ADA, 2018; SBD, 2016). Dentre as doenças cardiovasculares, a aterosclerose é a forma mais frequente e a mais grave. A dislipidemia, o estresse oxidativo, a resistência à insulina, a disfunção endotelial, a microangiopatia diabética, a glicação não enzimática, as alterações homeostáticas, e as associações com outros fatores de risco são as principais causas relacionadas à fisiopatologia da aterosclerose no diabetes (SBD, 2016; PARK et al., 2016). Nas dislipidemias é comum encontrar nos indivíduos com DM2 a hipertrigliceridemia, associada à redução de (HDL-colesterol e o aumento no número de partículas de LDL pequenas e densas (CRAWFORD, 2017). Um dos mecanismos patogênicos que explica o aumento do risco de aterosclerose e doença coronariana no diabetes é o desequilíbrio entre oxidantes e antioxidantes em favor dos oxidantes, ocasionadas pelo estresse 
oxidativo (SALAU, ERUKAINURE, ISLAM; 2020. A excessiva produção de radicais livres e espécies reativas de oxigênio (EROs) está relacionada com danos celulares a macromoléculas biológicas como lipídios, proteínas, DNA e carboidratos (KLEIN et al., 2011). A hiperglicemia resulta da oxidação da glicose, glicação não enzimática e disfunção monocítica, que conduz para o aumento na produção de radicais livres. Isso é agravado pela diminuição dos níveis de antioxidantes levando ao dano oxidativo, demonstrado por altos níveis de produtos de peroxidação lipídica e de DNA encontrado em pacientes com DM2 (ULLAH, 2016).

O principal objetivo do controle da hiperglicemia é a prevenção do desenvolvimento das complicações diabéticas e a redução final nos custos financeiros para os tratamentos dos agravamentos da doença. A maioria dos pacientes DM1 faz o controle glicêmico através da utilização de insulina, e o DM2 através da medicação hipoglicemiante, mas são enfatizadas as associações com exercícios físicos e planos nutricionais, juntamente com as terapias hipoglicêmicas (FRANCO, 2018).

\section{TERAPIAS NUTRICIONAIS E QUALIDADE DE VIDA}

No presente estudo, o consumo do araçá vermelho por 60 dias por pacientes com DM2 foi responsável por reduzir em aproximadamente $25 \%$ os índices de glicemia de jejum, quando comparado com os níveis basais (Tabela 3), comportamento não observado nos pacientes pertencentes ao grupo controle.

Tabela 3: Perfil glicêmico de indivíduos com DM2 no período basal e após 60 dias de ingestão de araçá vermelho.

\begin{tabular}{cccc}
\hline & $\begin{array}{c}\text { Tempo } \\
\text { (dias) }\end{array}$ & $\begin{array}{c}\text { Intervençåo } \\
(\mathbf{n}=\mathbf{1 3})\end{array}$ & $\begin{array}{c}\text { Controle } \\
(\mathbf{n}=\mathbf{9})\end{array}$ \\
\hline GLICEMIA (mg.dL ${ }^{-1}$ ) & 1 & $217,5 \pm 26,8$ & $143,9 \pm 16,8 \S$ \\
& 60 & $161,8 \pm 14,3^{*}$ & $140,3 \pm 15,2 \mathrm{~b}$ \\
\hline${ }^{*} \mathrm{p}<0,05 ;{ }^{*}$ comparado ao respectivo valor basal (Teste $t$ pareado de Student). $\S \mathrm{p}<0,05$ comparado
\end{tabular}
entre grupos diferentes (Teste t não pareado) representam diferença significativa entre os valores.

Esta redução está associada à menores índices de complicações do diabetes como risco de infarto agudo do miocárdio e risco de complicações microvasculares decorrentes do diabetes (KLEIN et al., 2011). Esses resultados são explicados devido à presença dos flavonoides e ácidos fenólicos nos frutos de araçá vermelho, que apresentam efeito benéfico à saúde por meio de diversos mecanismos, dentre eles ação antioxidante (PEREIRA et al., 2018). Os mecanismos antidiabético dos compostos fenólicos são atribuídos principalmente à sua capacidade em eliminar os radicais livres gerados pela hiperglicemia bem como impedir outras atividades mediadas por oxidação (SALAU, ERUKAINURE, ISLAM; 2020).

Com relação a qualidade de vida dos pacientes, observou-se uma diferença estatística na pontuação total do instrumento entre os grupos controle e intervenção e no domínio: Preocupações relacionadas ao diabetes. O grupo intervenção apresentou uma melhor pontuação, evidenciando uma melhor qualidade de vida (Tabela 4). 
Tabela 4 - Resultados da média por domínio e total do DQOL-Brasil

\begin{tabular}{cccc}
\hline \multirow{2}{*}{ DQOL } & $\begin{array}{c}\text { Controle } \\
(\mathbf{n}=\mathbf{0 9})\end{array}$ & $\begin{array}{c}\text { Intervenção } \\
(\mathbf{n}=\mathbf{1 3})\end{array}$ & \\
\cline { 2 - 4 } & Média (EP) & Média (EP) & $\mathbf{p}$ \\
\hline Satisfação & $2,51(0,56)$ & $2,44(0,65)$ & 0,8 \\
\hline Impacto & $2,40(0,87)$ & $1,88(0,35)$ & $0,06^{\star}$ \\
\hline $\begin{array}{c}\text { cupaçöes Sociais Vocacionais } \\
\text { Preocupações relacionadas ao } \\
\text { diabetes }\end{array}$ & $\mathbf{1 , 3 5 ( 0 , 5 4 )}$ & $\mathbf{1 , 2 6 ( 0 , 5 0 )}$ & 0,69 \\
\hline Total & $2,81(0,82)$ & $2,02(0,88)$ & $0,05^{\star}$ \\
\hline
\end{tabular}

${ }^{*} p<0,05$; comparado entre grupos diferentes (Teste t não pareado) representam diferença significativa entre os valores.

Foi identificada nesse estudo um efeito positivo entre o consumo de araçá vermelho, melhora nos níveis glicêmicos e melhor qualidade de vida relatada. Os resultados da avaliação de qualidade de vida, após intervenção, apontaram um índice substancialmente melhor no grupo intervenção. A população estudada foi predominante feminina, no entanto, o estudo de validação DQOLBrasil apresentou característica semelhante (CORRER et al. 2008). Os indivíduos do grupo intervenção pontuaram melhor o domínio impacto, o que implica em uma menor relação da doença com a qualidade de vida. Ao analisar o domínio de preocupações relacionadas ao diabetes, observou-se que os participantes do grupo controle apresentam maior preocupação com a DM e suas complicações do que o grupo intervenção. Acredita-se que essa preocupação resulte em maior sofrimento, o que afeta diretamente a QV.

O grupo intervenção apresentou melhor QV total. Este mesmo grupo também apresenta um percentual maior de indivíduos casados. A melhor adaptação ao diabetes tem sido relacionada ao suporte familiar (AUGUST et al., 2013), assim como a melhor adaptação ao tratamento tem sido vinculada a este estado civil (TAVARES et al., 2007).

\section{CONSIDERAÇÕES FINAIS}

Os resultados do presente estudo atestam uma melhora nos parâmetros glicêmicos do grupo intervenção que consumiu araçá vermelho no período de 60 dias. Ao avaliar a qualidade de vida dos participantes, percebe-se que 0 grupo intervenção teve uma pontuação significativamente melhor que o grupo controle, que não fez uso do fruto. Percebe-se que o grupo controle também apresenta maior preocupação com complicações causadas pela doença. Sugere-se, a partir dos dados aqui identificados, mais estudos de intervenção relacionando tratamento não medicamentoso, terapia nutricional e qualidade de vida relacionada à saúde.

\section{REFERÊNCIAS}

AMERICAN DIABETES ASSOCIATION. Standards of medical care in diabetes2018. Diabetes Care, v. 33, p. 11-61, 2010. 
AUGUST, et al. Spouses' involvement in their partners' diabetes management: Associations with spouse stress and perceived marital quality. J Fam Psychol, v. 27, p. 712-721, 2013.

CORRER, C. J. et al. Tradução para o Português e Validação do Instrumento Diabetes Quality of Life Measure (DQOL-Brasil). Arq Bras Endrocrinol Metab, v. 52, p. 515-522, 2008.

CRAWFORD, K. Review of 2017 Diabetes standards of care. Nurs Clin N Am, v. 52, 621-663, 2017.

KLEIN, G.A. et al. Mate Tea (Ilex paraguariensis) Improves glycemic and lipid profiles of Type 2 Diabetes and Pre- Diabetes Individuals : A Pilot Study. Journal of the American College of Nutrition, v. 30, p.37-41, 2011.

LIMA, A. C. S.; ARAÚJO, M. F. M.; DE FREITAS, R. W. J. F.; ZANETTI, M. L.; ALMEIDA, P. C.; DAMASCENO, M. M. C. Fatores de risco para diabetes mellitus tipo 2 em universitários: associação com variáveis sociodemográficas. Rev. Latino-Am. Enfermagem. v. 22, p. 484-90, 2014.

PARK, G. M. et al. Family history of diabetes and the risk of subclinical atherosclerosis. Diabetes \& Metabolism, v. 42, 170-177, 2016.

PEREIRA, et al. Psidium cattleianum fruits: A review on its composition and bioactivity. Food Chem. v. 258, p.95-103, 2018.

TAVARES, et al. Caracterização de idosos diabéticos atendidos na atenção secundária. Cien Saude Colet, v. 12, p. 1341-1352, 2007.

TRIKKALINOU A, PAPAZAFIROPOULOU AK, MELIDONIS A. Type 2 diabetes and quality of life. World J Diabetes. v. 8, p. 120-129, 2017.

The WHOQOL Group. The World Health Organization quality of life assessment (WHOQOL): position paper from the World Health Organization. Soc Sci Med 1995; 41:1403-10.

SALAU, V. F., ERUKAINURE, O. L., ISLAM, S. Chapter 29 - Phenolics: therapeutic applications against oxidative injury in obesity and type 2 diabetes pathology. In: Pathology Oxidative Stress and Dietary Antioxidants, p. 297-307. Academic Press Books - Elsevier: Cambridge, Massachusetts, EUA, 2020.

SBD. Classificação Etiológica - Diretrizes da Sociedade Brasileira de Diabetes 2016. Disponível em: http://www.diabetes.org.br/politicas/diretrizesonline.php. Acesso em: 07 abril.2021.

ULLAH, A.; KHAN, A.; KHAN, I. Diabetes mellitus and oxidative stress-A concise Review. Saudi Pharmaceutical Journal, v. 24, 547-553, 2016. 